\title{
Acute portal vein thrombosis secondary to hyperhomocysteinemia with folic acid deficiency and methyl tetrahydrofolate reductase mutation: a case report and literature review
}

Yosra Messaoudi ${ }^{1}$, Mohamed Hedfi ${ }^{2}$, Najeh Benhalima ${ }^{1}$, Adnen Chouchene ${ }^{2}$

${ }^{1}$ Department of Cardiology, Kairouan Hospital, Kairouan, Tunisia ${ }^{2}$ Department of Surgery, FSI Hospital Tunis, Tunis, Tunisia

Submitted: 19 March 2016

Accepted: 15 April 2016

Arch Med Sci Atheroscler Dis 2016; 1: e1-e5 DOI: 10.5114/amsad.2016.59575

Copyright (c) 2016 Termedia \& Banach

Acute portal vein thrombosis (PVT) is an uncommon but serious cause of abdominal pain, typically associated with cirrhosis. However, it might also occur in the absence of liver disease. In such cases, an underlying thrombotic disorder has to be suspected and investigated. Hyperhomocysteinemia has been well documented to increase the risk of arterial thrombotic events [1], peripheral arterial disease [2] and stroke [3].

It is also a risk factor for deep-vein thrombosis in the general population $[4,5]$. Association with portal vein thrombosis is very unusual, and only a few cases have been reported.

We present a case of acute thrombosis of the portal vein in a patient with hyperhomocysteinemia associated with low serum folate levels and a methyl tetrahydrofolate reductase mutation.

The case report is followed by a discussion of the risk factors and etiology, the clinical presentations of PVT, as well as a review of the investigations and management proposed in the literature.

A 57-year-old man presented to the emergency department complaining of abdominal pain that had lasted for 3 days. The patient was a heavy smoker. His medical history was unremarkable. Specifically he had no history of liver disease and he was on no medications. On examination, he was icteric and was febrile at $38.2^{\circ} \mathrm{C}$. His blood pressure was $130 / 70 \mathrm{~mm} \mathrm{Hg}$ and his heart rate was 100 beats/min. Abdominal palpation revealed tenderness in the right upper quadrant, without peritoneal signs. Laboratory data showed a white blood cell count of $13500 / \mathrm{mm}^{3}$, aspartate aminotransferase and alanine aminotransferase 4 times higher than normal values, alkaline phosphatase of $500 \mathrm{U} / \mathrm{l}$ (normal: 15250), $\gamma$-glutamyltransferase of $120 \mathrm{U} / \mathrm{l}$ (normal 8-61), direct bilirubin of $35 \mathrm{mg} / \mathrm{dl}$ (normal 0-0.25), total bilirubin of $55 \mathrm{mg} / \mathrm{dl}$ (normal 0.2-1.2), C-reactive protein of $60 \mathrm{mg} / \mathrm{l}$, and hemoglobin of $12.4 \mathrm{~g} / \mathrm{dl}$.

Abdominal ultrasonography revealed a tubular hypoechoic lesion in the portal vein with no blood flow at the lesion site (Figure 1). The liver and the bile ducts appeared normal. The abdominal computed tomography (CT) scan (Figure 2) did not show extension to the mesenteric venous arches or the splenic vein. There was no collateral circulation or cavernoma.

\author{
Corresponding author: \\ Mohamed Hedfi \\ FSI Hospital \\ Marsa 2073, Tunisia \\ Phone: +21698825320 \\ Fax: +21670213456 \\ E-mail: hedfi2007@yahoo.fr
}

$$
\text { cavernoma. }
$$




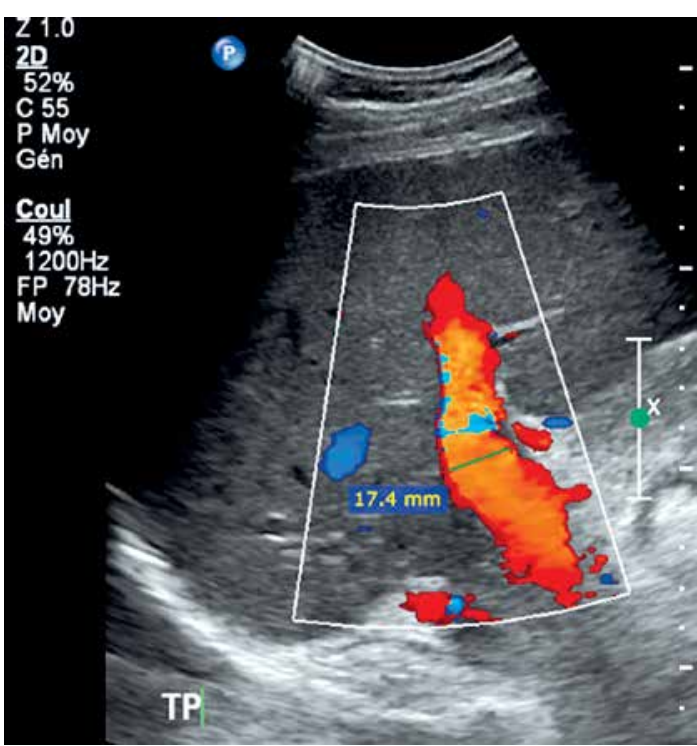

Figure 1. Ultrasonography showed a dilated and obstructed portal vein

The patient was diagnosed with acute PVT and was placed on anticoagulant therapy with low-molecular-weight heparin and acenocoumarol with a target INR of 2-2.5.

An extensive coagulation work-up showed a normal prothrombin time, normal platelet count, and normal activated partial thromboplastin time.

Prothrombotic disorders were researched - protein S, protein C, factor V Leiden mutation, factor II mutation and anti-thrombin III deficiency - but none were found.

Immunoglobulin $\mathrm{G}$ and IgM assays for anti-cardiolipin were negative. Lupus anti-coagulant was also negative, and so were antinuclear antigen and anti-double-stranded DNA. Also, there was no evidence of Behçet's disease.

However, he had hyperhomocysteinemia reaching $45 \mu \mathrm{mol} / \mathrm{l}(\mathrm{N}<15)$. The vitamin $\mathrm{B}_{12}$ concentration was normal. His serum folate was low: $3.6 \mathrm{nmol} / \mathrm{l}(\mathrm{N}: 6-35 \mathrm{nmol} / \mathrm{l})$.

A hematological consultation advised looking for Janus kinase (JAK)-2 gene mutation; this was negative, suggesting the absence of a myeloproliferative disorder.

Genetic aberrations in the cystathionine $\beta$-synthase (CBS) and methylenetetrahydrofolate reductase (MTHFR) genes were studied.

The search for polymorphism of methyl-tetrahydrofolate reductase (MTHFR) after amplification by enzymatic digestion of DNA showed the presence of the C677T mutation in the homozygous state.

Therefore the patient was started on vitamin supplementation: folate $(10 \mathrm{mg} /$ day $)$, vitamin $B_{6}$ (750 mg/day), vitamin $B_{12}(1000 \mu \mathrm{g} /$ day).

His condition improved significantly, and three months after initiation of therapy (anticoagulant treatment and vitamin) the homocysteine level

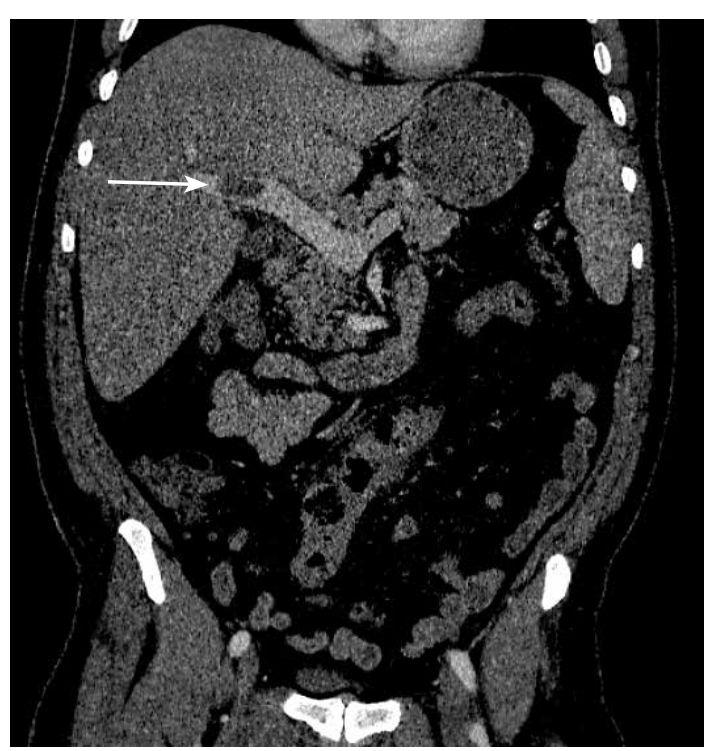

Figure 2. Computed tomographic scan of the abdomen showing a portal vein thrombus

was normal. Follow-up ultrasonography confirmed the disappearance of the thrombus.

Future monitoring was based on regular measurement of homocysteine and folate supplementation on demand at every elevation of homocysteine above normal values.

Portal vein thrombosis is a complete or partial obstruction of blood flow in the portal vein, due to the presence of a thrombus in the vessel lumen [6]. The thrombosis develops in the trunk of the portal vein and can include its right and left intrahepatic branches. It may extend to the splenic or superior mesenteric veins.

In the general population PVT is thought to be rare, with a prevalence of $1 \%$ [7].

Nevertheless, its prevalence among cirrhotic patients varies between $4.4 \%$ and $15 \%$, and it is responsible for about $5-10 \%$ of all cases of portal hypertension [8].

Portal vein thrombosis can be acute or chronic. Studies have generally considered PVT to be acute if symptoms develop less than 60 days before the diagnosis. However, this definition is not accepted by all the authors $[9,10]$. Chronic PVT presents with the complications of portal hypertension: splenomegaly or hypersplenism and variceal bleeding. It may also be asymptomatic and discovered incidentally on imaging.

Our patient developed acute spontaneous PVT. In fact, the clinical presentation depends on the rapidity and extent of the thrombosis.

Acute PVT is characterized by abdominal pain and nausea. It may also be asymptomatic. When the PVT extends to the mesenteric or splenic veins, signs of ischemia and sepsis such as shock or multiorgan failure may occur. That is why diagnosis based on clinical findings is often challenging. 
In cases of suspected PVT, the initial laboratory tests should include at least liver parameters as well as infection, kidney, and basic coagulation values.

Abdominal color Doppler ultrasound (US) is the investigation of choice, with a sensitivity and specificity between $60 \%$ and $100 \%[11,12]$. However, US is less reliable in determining the extension of the thrombus to the mesenteric circulation [13]. Computed tomography scanning and magnetic resonance imaging can easily obtain this information. Contrast-enhanced computed tomography scan with the portal and arterial phase is the imaging technique of choice. It is very useful for identification of the possible cause and potential complications, such as bowel ischemia and perforation [14].

When the diagnosis of acute PVT is made, an immediate start of anticoagulation therapy is needed. We prefer to use unfractionated heparin. Also, low-molecular-weight heparin (LMWH) may be used. The main goal of this treatment is to achieve recanalization of the portal vein or at least to prevent thrombus extension. In fact, the recanalization rate of the main portal trunk and its left or right branch after anticoagulant therapy is estimated at 39\% [15].

In general, 3 to 6 months of anticoagulation therapy is proposed by most authors [16]. In the case of hypercoagulopathy, lifelong anticoagulation is usually recommended.

However, there is no consensus on the duration and medication of anticoagulation.

Other therapeutic options are currently available: Local or systemic thrombolytic therapy is indicated in patients with symptomatic acute PVT but without signs of bowel ischemia. However, numerous complications have been described, in particular bleeding, and they are present in up to $60 \%$ of patients [17].

Unfortunately, no prospective study has been performed to compare anticoagulation and thrombolytic treatment alone.

Surgical thrombectomy is performed only if the patient undergoes laparotomy for acute abdomen or intestinal ischemia.

Mechanical thrombectomy via a transhepatic or transjugular approach is another therapeutic option. It has the advantage of rapid removal of the thrombus [18, 19].

Lang et al. proposed an algorithm for the management of acute PVT depending on the clinical presentation [20] (Figure 3).

Some authors also recommend immediate initiation of broad-spectrum antibiotic therapy, because bacteremia is associated with PVT [21].

A variety of situations have been described to cause portal vein thrombosis, such as cirrhosis, malignancies and infection of the gastrointestinal tract. In all cases of portal vein thrombosis when no overt cause is found, coagulopathy should be ruled out. In fact, a multitude of thrombophilic conditions have been described in the literature. These prothrombotic states can be inherited or acquired (Table I) [22]. Among them, factor $\mathrm{V}$ Leiden mutations and myeloproliferative disorders are most commonly associated with PVT.

Hyperhomocysteinemia is an independent risk factor for thromboembolism even at mild (15$20 \mu \mathrm{mol} / \mathrm{l})$ and moderate $(25-50 \mu \mathrm{mol} / \mathrm{l})$ levels [23]. It causes a hypercoagulable state by increasing platelet aggregation, activation of factor $\mathrm{V}$, $\mathrm{X}$, and XII and inhibition of antithrombin III and protein $C$. Metabolism of homocysteine requires folate and vitamin $B_{12}$ or vitamin $B_{6}$. Thus elevated homocysteine levels can result from low levels of folic acid, vitamin $\mathrm{B}_{6}$ or vitamin $\mathrm{B}_{12}$. Moreover, several genetic alterations in enzymes involved in homocysteine metabolism have been mentioned. The more severe cases of hyperhomocysteinemia are caused by homozygous defects in genes encoding for enzymes of homocysteine metabolism. The classic form "congenital homocystinuria" is caused by homozygous defects in the gene encoding for cystathionine $\beta$-synthase. In these patients, plasma homocysteine concentrations can be as high as $400 \mu \mathrm{mol} / \mathrm{l}$ [24]. This disease is characterized by early arteriosclerosis, thromboembolic complications, ectopia lentis, skeletal abnormalities and mental retardation. Moderate hyperhomocysteinemia is more common than severe elevation and may be caused by less severe defects in genes encoding for enzymes or from inadequate status of vitamins involved in homocysteine metabolism. The most common genetic abnormality observed in the general population in the homozygous state, with a frequency of $1.4 \%$ to $15 \%$ depending on regions, is the C677T mutation of the gene MTHFR, causing a variant of MTHFR that was distinguished from the normal enzyme by its lower specific activity (50\%) and its thermolability $[25,26]$.

The impact of the MTHFR thermolabile variant on plasma homocysteine levels is however not clear. Kang et al. suggested that the phenotypic expression of the MTHFR genotypes is dependent on the availability of folate, suggesting that homozygotes for the thermolabile genotype might have a higher folate requirement than individuals with a normal genotype $[27,28]$.

An inverse correlation between homocysteine and folate or vitamin $B_{12}$ plasma concentrations and the efficacy of vitamin supplementation in the lowering of plasma homocysteine levels has been demonstrated.

Patients with portal vein thrombosis and hyperhomocysteinemia have rarely been reported. Like our case, Tan et al. reported a case of portal vein thrombosis caused by hyperhomocysteinemia that led to bowel gangrene, and was corrected 


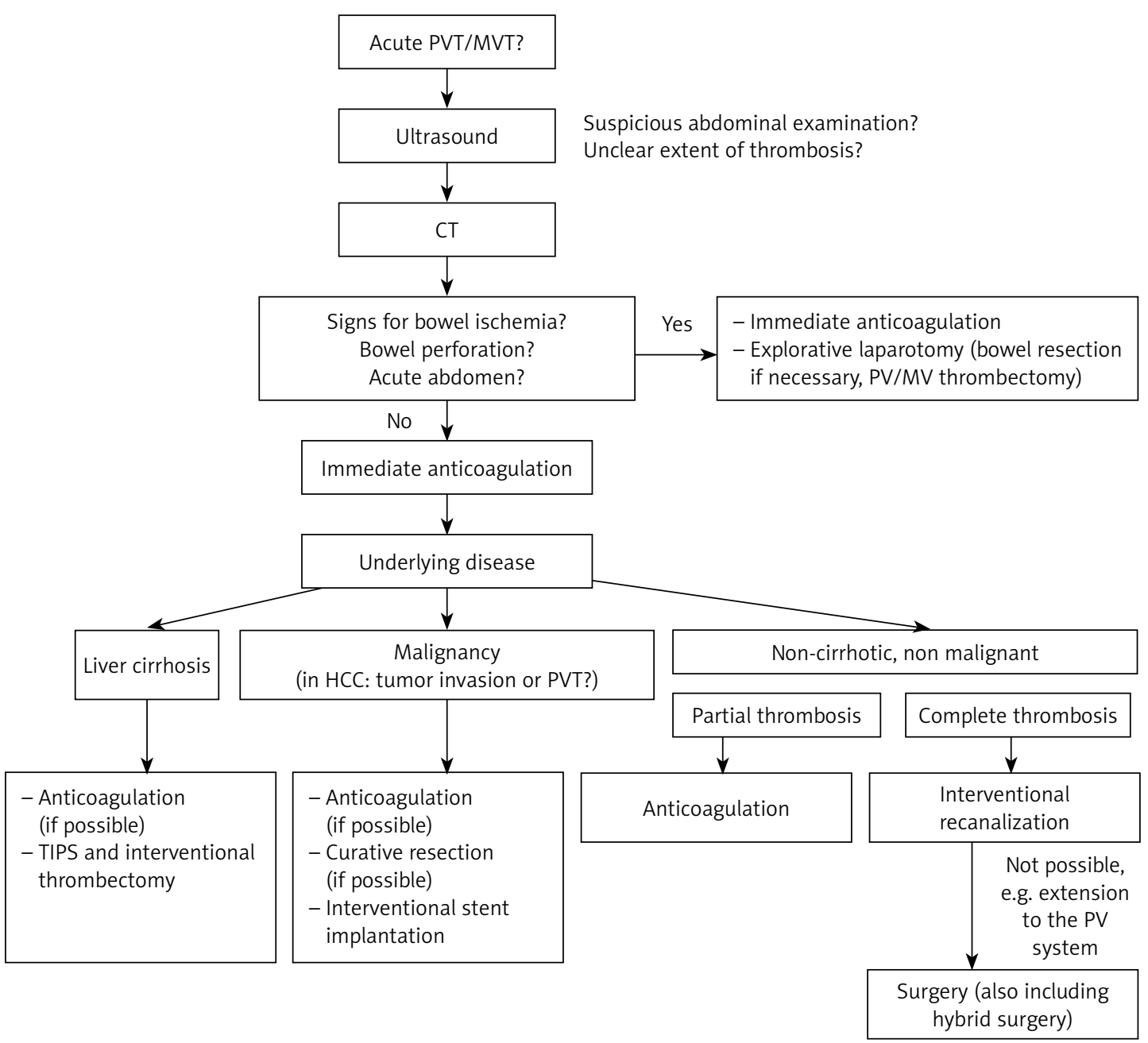

Figure 3. Proposed diagnostic and therapeutic algorithm for patients with acute portal vein thrombosis/mesenteric vein thrombosis (PVT/MVT)

Table I. Prevalence of thrombotic risk factors in patients with acute or chronic PVT

\begin{tabular}{|lc|}
\hline Risk factor & PVT patients (\%) \\
\hline Myeloproliferative disorders: & $30-40$ \\
\hline Atypical & 14 \\
\hline Classical & 17 \\
\hline Antithrombin deficiency & $0-26$ \\
\hline Protein C deficiency & $0-26$ \\
\hline Protein S deficiency & $2-30$ \\
\hline Factor V Leiden mutation & $6-32$ \\
\hline Prothrombin mutation & $14-40$ \\
\hline $\begin{array}{l}\text { TT677 methylene tetrahydrofolate } \\
\text { reductase (MTHFR) genotype }\end{array}$ & $11-50$ \\
\hline Antiphospholipid syndrome & $6-19$ \\
\hline Hyperhomocysteinemia & $12-22$ \\
\hline Recent pregnancy & $6-40$ \\
\hline Recent oral contraceptive use & 12 \\
\hline
\end{tabular}

with folate and vitamin $\mathrm{B}_{6}$ supplementation [29]. Kocher et al. found hyperhomocysteinemia to be present in $12 \%$ of patients presenting with a prior diagnosis of idiopathic PVT [30].

It remains unclear however whether hyperhomocysteinemia of different causes leads to the same risk of thrombosis. But, it is well known that vitamin supplementation lowers homocysteine concentrations in almost all subjects with hyperhomocysteinemia, regardless of the underlying cause.

In conclusion, PVT is an uncommon cause of abdominal pain, and in the absence of hepatic disease, an underlying thrombophilia should be suspected. We emphasize the importance of measuring serum homocysteine in all patients with PVT to avoid missing this rare but curable condition. Management of acute PVT is complex because of the lack of evidence-based therapeutic algorithms. Close cooperation between surgery, radiology and internal medicine is crucial.

\section{Conflict of interest}

The authors declare no conflict of interest. 


\section{References}

1. Stampfer MJ, Malinow MR, Willett WC, Newcomer LM, Upson B, Ullmann D, et al. A prospective study of plasma homocysteine and risk of myocardial infarction in US physicians. JAMA 1992; 268: 877-81.

2. Khandanpour N, Loke YK, Meyer FJ, Jennings B, Armon MP. Homocysteine and peripheral arterial disease: systematic review and meta-analysis. Eur J Vasc Endovasc Surg 2009; 38: 316-22.

3. Towfighi A, Markovic D, Ovbiagele B. Pronounced association of elevated serum homocysteine with stroke in subgroups of individuals: a nationwide study. J Neurol Sci 2010; 298: 153-57.

4. Köktürk N, Kanbay A, Aydoğdu M, Özyılmaz E, Bukan N, Ekim N. Hyperhomocysteinemia prevalence among patients with venous thromboembolism. Clin Appl Thromb Hemost 2011; 17: 487-93

5. Cattaneo M. Hyperhomocysteinemia and venous thromboembolism. Semin Thromb Hemost 2006; 32: 716-23.

6. Bayraktar Y, Harmanci O. Etiology and consequences of thrombosis in abdominal vessels. World J Gastroenterol 2006; 12: 1165-74.

7. Ogren $M$, Bergqvist D, Bjorck $M$, et al. Portal vein thrombosis: prevalence, patient characteristics and lifetime risk: a population study based on 23,796 consecutive autopsies. World J Gastroenterol 2006; 12: 2115-9.

8. Amitrano L, Guardascione MA, Brancaccio V, et al. Risk factors and clinical presentation of portal vein thrombosis in patients with liver cirrhosis. J Hepatol 2004; 40: 736-41.

9. Ponziani FR, Zocco MA, Campanale C, et al. Portal vein thrombosis: insight into physiopathology, diagnosis, and treatment. World J Gastroenterol 2010; 16: 143-55.

10. Liappis AP, Roberts AD, Schwartz AM, et al. Thrombosis and infection: a case of transient anti-cardiolipin antibody associated with pylephlebitis. Am J Med Sci 2003; 325: 365-8.

11. Chawla Y, Duseja A, Dhiman RK. Review article: the modern management of portal vein thrombosis. Aliment Pharmacol Ther 2009; 30: 881-94.

12. Berzigotti A, Garcia-Criado A, Darnell A, Garcia-Pagan JC. Imaging in clinical decision-making for portal vein thrombosis. Nat Rev Gastroenterol Hepatol 2014; 11: 308-16.

13. Webster GJ, Burroughs AK, Riordan SM. Review article: portal vein thrombosis - new insights into etiology and management. Aliment Pharmacol Ther 2005; 21: 1-9.

14. Kocher G, Himmelmann A. Portal vein thrombosis (PVT): a study of 20 non-cirrhotic cases. Swiss Med Wkly 2005; 135: 372-6.

15. Plessier A, Darwish-Murad S, Hernandez-Guerra M, et al. Acute portal vein thrombosis unrelated to cirrhosis: a prospective multicenter follow-up study. Hepatology 2010; 51: 210-8.

16. Handa P, Crowther M, Douketis JD. Portal vein thrombosis: a clinician-oriented and practical review. Clin App Thromb Hemost 2013; 20: 498-506.

17. Hollingshead M, Burke CT, Mauro MA, Weeks SM, Dixon RG, Jaques PF. Transcatheter thrombolytic therapy for acute mesenteric and portal vein thrombosis. J Vasc Interv Radiol 2005; 16: 651-61.

18. Hall TC, Garcea G, Metcalfe M, Bilku D, Dennison AR Management of acute non-cirrhotic and non-malig nant portal vein thrombosis: a systematic review. World J Surg 2011; 35: 2510-20.

19. Jun KW, Kim MH, Park KM, et al. Mechanical thrombectomy-assisted thrombolysis for acute symptomatic portal and superior mesenteric venous thrombosis. Ann Surg Treat Res 2014; 86: 334-41.

20. Lang SA, Loss M, Wohlgemuth WA, Schlitt HJ. Clinical management of acute portal/mesenteric vein thrombosis. Viszeralmedizin 2014; 30: 394-400.

21. Ni YH, Wang NC, Peng MY, Chou YY, Chang FY. Bacteroides fragilis bacteremia associated with portal vein and superior mesentery vein thrombosis secondary to antithrombin III and protein C deficiency: a case report. J Microbiol Immunol Infect 2002; 35: 255-8.

22. DeLeve LD, Valla DC, Garcia-Tsao G. Vascular disorders of the liver. Hepatology 2009; 49: 1729-64.

23. Carmel R, Green R, Rosenblatt DS, et al. Update on cobalamin, folate, and homocysteine. Hematology Am Soc Hematol Educ Program 2003; 1: 62-81.

24. D'Angelo A, Selhub J. Homocysteine and thrombotic disease. Blood 1997; 90: 1-11.

25. Upchurch GR Jr, Welch GN, Freedman JE, Loscalzo J. Homocysteine attenuates endothelial glutathione peroxidase and thereby potentiates peroxide-mediated injury. Circulation 1995; 92: 1228.

26. Goyette P, Sumner JS, Milos R, et al. Human methylenetetrahydrofolate reductase: isolation of cDNA, mapping and mutation identification. Nat Genet 1994; 7: 95-200.

27. Kang SS, Zhou J, Wong PWK, Kowalisyn J, Strokosch G. Intermediate homocysteinemia: a thermolabile variant of methylenetetrahydrofolate reductase. Am J Hum Genet 1988; 43: 414-21.

28. Selhub J, Jacques PF, Wilson PW, Rush D, Rosenberg IH. Vitamine status and intake as primary determinants of homocysteinemia in an elderly population. JAMA 1993; 270: 2693-8.

29. Tan K, Chow PKH, Tan YM, Thng CH. Portal vein thrombosis secondary to hyperhomocysteinemia: a case report. Dig Dis Sci 2006; 51: 1218-20.

30. Kocher G, Himmelman A. Portal vein thrombosis: a study of 20 non cirrhotic cases. Swiss Med Wkly 2005; 135: $372-6$. 